\title{
Análisis de las propiedades psicométricas de un cuestionario para medir la intensidad de la molestia ante la mentira en la relación de parejas juveniles mediante el modelo Rasch
}

\author{
Gabriel Yánez Canal ${ }^{1}$, Humberto Yánez Canal ${ }^{2}$, Fernando Robert Ferrel Ortega ${ }^{3}$ \\ Universidad Industrial de Santander-Colombia ${ }^{1}$, Universidad Cooperativa de \\ Colombia ${ }^{2}$,Universidad del Magdalena-Colombia ${ }^{3}$
}

En este artículo se presenta el análisis de las propiedades psicométricas de un cuestionario elaborado para conocer el nivel de intensidad de la molestia que sienten jóvenes heterosexuales ante la mentira de sus parejas, realizado mediante el modelo Rasch. El test está compuesto por 15 ítems y se aplicó a 503 estudiantes en cursos universitarios de pregrado, con edades entre 16 y 22 ańos, de diferente sexo y con experiencia en relaciones de pareja. Los resultados muestran el buen ajuste de los datos al modelo, garantizando la validez del cuestionario. Si bien el test inicial está bien construido y es compatible con la mayoría de los niveles de los estudiantes encuestados, se podrían diseñar nuevos ítems para ampliar el rango de medidas de la molestia ante la mentira y con ello aumentar su validez.

Palabras clave: mentira, intensidad de la molestia ante la mentira, modelo Rasch, cuestionario, propiedades psicométricas.

Analysis of the psychometric properties of a questionnaire to measure the intensity of discomfort due to lying in the relationship of young couples using the Rasch model

This article presents an analysis using the Rasch model of the psychometric properties of a questionnaire elaborated to measure the level of intensity of the discomfort that young heterosexuals feel due to a partner's lie. The test consists of 15 items and was administered to 503 male and female students in university undergraduate courses between the ages of 16 and 22, who had experience in relationships. The results show the good fit of the data to the model, guaranteeing the validity of the questionnaire. Although the initial test is well-constructed and compatible with most of the levels of the students surveyed, new items

1 Doctor en Matemáticas. Docente titular a tiempo completo en la Universidad Industrial de Santander (UIS). Colombia. Dirección postal: Ciudad Universitaria, Cra. 27 \#9, Bucaramanga, Santander, Colombia. Contacto: gyanez@uis.edu.co. https://orcid. org/0000-0001-5538-074X

2 Doctor en Democracia, Paz y Conflicto. Docente investigador de tiempo completo en la Universidad Cooperativa de Colombia sede Santa Marta, Colombia. Dirección postal: Calle 30, Santa Marta, Magdalena, Colombia. Contacto: humberto.yańez@campusucc.edu.co. https://orcid.org/0000-0003-0127-7880

3 Doctor en Ciencias de la Educación. Docente investigador de tiempo completo en la Universidad Cooperativa de Colombia sede Santa Marta, Colombia. Dirección postal: Calle 30, Santa Marta, Magdalena, Colombia. Contacto: fernando.ferrel@campusucc.edu.co. https:// orcid.org/0000-0001-7411-3516 
could be designed to extend the range of measures of discomfort and thereby increase the instrument's validity.

Keywords: Lie, intensity of discomfort before lying, Rasch model, questionnaire, psychometric properties.

Análise das propriedades psicométricas de um questionário para medir a intensidade do mal-estar em relaçáo à mentira em casais jovens mediante o modelo Rasch

Este artigo apresenta a análise realizada com o modelo Rasch, das propriedades psicométricas de um questionário desenvolvido para conhecer o nível de intensidade de mal-estar que jovens heterossexuais sentem em relação às mentiras de seus parceiros. $\mathrm{O}$ teste é composto por 15 itens e foi aplicado a 503 estudantes universitários de graduaçáo, com idades entre os 16 e 22 anos, de ambos sexos e com experiência em relacionamentos de casal. Os resultados mostram um bom ajuste dos dados ao modelo, garantindo a validade do questionário. Embora o teste inicial seja bem construído e compatível com a maioria dos níveis dos estudantes pesquisados, novos itens podem ser desenvolvidos para ampliar a gama de medidas de mal-estar relação à mentira e, assim, aumentar a validade do questionário.

Palavras-chave: mentira, intensidade do mal-estar em relação à mentira, modelo Rasch, questionário, propriedades psicométricas

Analyse des propriétés psychométriques d'un questionnaire pour mesurer l'intensité des nuisances avant de mentir dans la relation de jeunes couples à l'aide du modèle de Rasch

Cet article présente l'analyse des propriétés psychométriques d'un questionnaire élaboré pour connaître le niveau d'intensité de la gêne ressentie par les jeunes hétérosexuels devant le mensonge de leurs partenaires, réalisée selon le modèle de Rasch. Le test comprend 15 items et a été appliqué à 503 étudiants de 16 à 22 ans inscrits dans des cursus universitaires de premier cycle, de sexe différent et ayant une expérience des relations. Les résultats montrent le bon ajustement des données au modèle, garantissant la validité du questionnaire. Bien que le test initial soit bien construit et compatible avec la plupart des niveaux des étudiants interrogés, de nouveaux éléments pourraient être conçus pour élargir la gamme de mesures de la gêne ressentie et en augmenter ainsi la validité.

Mots-clés: mensonge, intensité de l'inconfort avant de mentir, modèle de Rasch, questionnaire, propriétés psychométriques. 
La preocupación sobre el impacto ético de la mentira y la verdad en las interacciones humanas ha generado diversas manifestaciones filosóficas desde tiempos remotos, particularmente en lo relacionado con la moral y el comportamiento social consecuente, tal como lo manifiestan Yáñez, Ferrel, Ortiz y Yańez (2017).

Nuevas investigaciones sobre la mentira y el engaño y sus efectos comportamentales muestran especial preocupación en la relación de pareja entre adultos jóvenes, la cual se vería afectada de distintas maneras poniéndola en crisis.

En efecto, se ha encontrado que debilitan la confianza en el otro y deterioran la totalidad de la relación poniendo en crisis su continuidad (Núñez, Cantó-Milà \& Seebach, 2015); que inciden como creencias en la generación de violencia en el noviazgo entre parejas de jóvenes universitarios, tanto directas (Botero, Builes, García-Bonilla, Gil-Saldarriaga \& Ramírez-Guerrero, 2016) como virtuales mediante el ciberacoso (Rodríguez, Martínez-Pecino \& Durán-Segura, 2015); y, que consideran al maltratador como psicópata o de personalidad antisocial, lo que tiende a patologizar la relación (Pozueco-Romero, Moreno-Manso, Blázquez-Alonso \& García-Baamonde, 2013).

Sin embargo, en una dirección distinta, se encuentran otros estudios que señalan que la mentira en la relación de noviazgo de parejas de jóvenes universitarios heterosexuales es común, pero no amenazan la estabilidad de la pareja ni generan violencia, por el contrario, en algunas ocasiones tienden a unir más a la pareja cuando éstos lo afrontan, conversan y llegan a acuerdos (Yánez et al., 2017).

En este trabajo, se asume la mentira como la práctica social que afecta más a uno de los miembros de la pareja, en cuanto contrasta sus supuestos morales, sus suposiciones fácticas y sus expectativas de conducta. 
A pesar de los resultados estos estudios, no se ha encontrado un instrumento válido para población colombiana que permita la medición de los efectos de la mentira en la relación de parejas jóvenes. Sólo se han hallado algunos estudios de validación de instrumentos sobre coerción sexual en parejas de universitarios, sobre violencia entre novios (Rodríguez, Martínez-Pecino \& Durán, 2010), pero no sobre los efectos de la mentira en la relación de pareja.

Por todo lo anterior, surgió la necesidad de buscar instrumentos validados para estudiar el impacto de la mentira en la relación de pareja en población colombiana, que permita su medición objetiva, cuyos resultados se presentan a continuación. Para ello, en el cuestionario aquí propuesto se buscó evaluar las diferentes intensidades y asociaciones subjetivas de la mentira, tanto en su reacción inmediata como de mediano y largo plazo, pues se considera que estas reacciones no son automáticas, sino que difieren en cada persona; sin embargo, el único ítem que mostró continuidad y desató mayores intensidades de molestia en este cuestionario fue el de infidelidad, cuya reacción fue la de "terminar" la relación.

En la dirección de estos dos factores se realizó la presente investigación, para conocer el efecto que tiene la mentira en las parejas de jóvenes universitarios. Este efecto se analizó en tres niveles: la intensidad de la molestia que se siente cuando la pareja miente, el efecto en la respuesta inmediata y el efecto sobre la relación de pareja en el mediano plazo. Los resultados relacionados con los dos últimos niveles se pueden consultar en Yáńez et al. (2017).

El análisis de los resultados de la aplicación del test, que son los que se analizan en este artículo, se realizó con el modelo Rasch, un modelo probabilístico que posee una serie de ventajas que los modelos de Teoría Clásica de Test (TCT) no poseen y que hacen que, a pesar de su sencillez, sea ampliamente utilizado (Wright \& Stone, 1979; Andrich, 1988; Hambleton, Swaminathan \& Rogers, 1991; Embretson \& Reise, 2000; Bond \& Fox, 2001).

Las ventajas del modelo Rasch están asociadas esencialmente a las siguientes propiedades: medición conjunta, independencia muestral u 
objetividad específica, medida lineal o de intervalo, y especificidad del error típico de medida (Prieto y Delgado, 2003).

1. Medición conjunta: El modelo Rasch evalúa la habilidad (cantidad del constructo que se quiere medir) de los individuos y la dificultad (cantidad del constructo que miden) de los ítems simultáneamente y en la misma medida (lógitos que no es otra cosa que el logaritmo de la razón de oportunidades. Odds ratio en inglés), lo que permite relacionar sus valores; más exactamente, evaluar la probabilidad de que un individuo de cierta habilidad seleccione una determinada respuesta cuando se enfrenta a un ítem de cierta dificultad y conocer cuáles ítems son más fáciles o difíciles para cada individuo. Esta característica del modelo permite elaborar ítems dirigidos a la población en estudio controlando de esta forma la validez del test.

2. Independencia muestral. Conocida también como separación de parámetros (Smith y Smith, 2004) u objetividad especifica como la denominaba Rasch (1977), se refiere a que las medidas obtenidas para las personas son independientes de la muestra de ítems utilizada. Igualmente, las medidas de los ítems son independientes de la muestra de personas a las cuales se les aplique el test. Esta independencia se manifiesta cuando se calcula la diferencia de las habilidades (dificultades) entre dos personas (ítems) y se observa que no depende del ítem (persona) que se toma como referencia para el cálculo de esa diferencia (Prieto y Delgado, 2003). Esta propiedad, que no poseen ni la TCT ni los modelos de Teoría de respuesta al ítem (TRI), permite comparar las medidas de personas, aunque hayan tomado test diferentes (Bond y Fox, 2001).

3. Medida lineal o de intervalo: las medidas en la escala construida poseen las propiedades de los números reales, en particular el hecho de que las diferencias tienen el mismo significado en toda la recta real, cosa que no ocurre con los puntajes que sin tratamiento alguno se asigna a las personas que responden un test. Esta propiedad es básica para realizar análisis estadísticos paramétricos con las medidas obtenidas por el modelo. 
4. Especificidad del error tipico de medida: A diferencia de la TCT el modelo Rasch genera medidas con sus respectivos errores. Estos errores en los ítems (personas) se estiman mejor con personas (ítems) que tengan medidas similares. Es decir, las medidas de los sujetos se estiman mejor con ítems de su misma medida: los de alto rendimiento con ítems difíciles y los de bajo rendimiento serán mejor estimadas con los ítems fáciles. Esta propiedad permite seleccionar los ítems, acorde con el nivel de los individuos que se quiere evaluar, generando mayor precisión en las medidas y, por lo tanto, aumentando la validez del test.

El modelo Rasch garantiza estas propiedades, siempre y cuando los datos se ajusten al modelo y se tenga unidimensionalidad en la medida, esto es, que representen un solo constructo (Bond y Fox, 2001). En consecuencia, parte importante del análisis que se muestra en este artículo está dirigido a validar estos supuestos que son garantía de validez (Smith y Smith, 2004), así como su nivel de confiabilidad para las personas y los ítems.

\section{El modelo de crédito parcial}

Existen varios tipos de modelo Rasch como respuesta a diferentes tipos de test. En nuestro caso, los ítems tienen formato Likert con 5 categorías que responden a diversos niveles de intensidad de la molestia ocasionada por la mentira siendo el modelo adecuado el de Escalas de Rendimiento (Rating Scale) cuya expresión algebraica para la probabilidad de que un sujeto $n$ de habilidad seleccione la categoría $x$ en el ítem $i$ de dificultad $H_{n}$ está dada por Andrich (1978):

$$
P_{n x i}=\frac{\exp \sum_{j=1}^{x}\left[H_{n}-\left(D_{i}+F_{x}\right)\right]}{\sum_{x=1}^{m+1} \exp \sum_{j=1}^{x}\left[H_{n}-\left(D_{i}+F_{x}\right)\right]} \quad x=1,2, \ldots m+1
$$

El número de categorías en la expresión (1) es $m+1$ que en nuestro caso corresponde al valor 5 . El valor $D_{i}+F_{x}$, determina el umbral que se corresponde con el punto en el cual la probabilidad de optar por la categoría $x$ es la misma que optar por la categoría $x$ - 1 cuando se 
enfrenta al ítem $i$. Obsérvese que los valores $F_{x}$ son iguales para todos los ítems, es decir, las distancias entre umbrales son siempre las mismas para todos los ítems. La dificultad $D_{i}$ del ítem se corresponde con el valor en el cual la probabilidad de seleccionar la categoría 1 es la misma que la de seleccionar la categoría $m+1$.

De la expresión (1) se deduce que la probabilidad de seleccionar una categoría superior en cualquier ítem es mayor en las personas con mayor habilidad, esto es, si una persona es más hábil (posee más cantidad del constructo medido) que otra, la probabilidad de que seleccione una categoría superior en cualquier ítem es siempre superior a la de la otra persona.

\section{Método}

\section{Participantes}

La población estuvo constituida por estudiantes universitarios matriculados en cursos universitarios de pregrado entre 16 y 22 ańos, de diferente sexo y con experiencia directa en relaciones de pareja.

La muestra se escogió utilizando un diseńo muestral estratificado proporcional al tamaño, con selección aleatoria de la población de estudiantes en el rango de edad considerado y matriculados en la Universidad Cooperativa de Colombia sede Santa Marta en el segundo semestre de 2014: 751 mujeres y 441 hombres de 5 Facultades (Medicina, Psicología, Ingeniería, Administración y Contaduría). El tamaño muestral se fijó en 546 estudiantes, distribuidos por sexo en proporción a su población. En el proceso de digitación se anularon 43 encuestas por errores en las respuestas, lo que redujo el número a 503. Los estudiantes firmaron un consentimiento informado que permitía a los investigadores utilizar la información recabada para propósitos investigativos.

\section{Medición}

En la Figura 1 se presenta el cuestionario para el registro de la información que permitió contextualizar de manera general la propuesta 
de estudio sobre los efectos de la mentira en las relaciones de los jóvenes en pareja.

Considerando la mentira en la relación de pareja como la práctica social que afecta más a uno de sus miembros poniéndola en crisis moral, se construyeron 15 ítems que presentaban situaciones, mensajes o acciones en tiempo verbal pretérito que implican mentira, simulación, apariencia, ocultamiento o engaño, escogidos después de un proceso de selección que implicó la validación por tres (3) jueces expertos en desarrollo juvenil, la aplicación de tres encuestas de pregunta abierta a una muestra de 150 estudiantes y, finalmente, con la aplicación de 4 pretest aplicados a varios grupos de 12 estudiantes.

Además, el proceso de elaboración del instrumento tuvo en cuenta referencias teóricas y explicaciones sobre las relaciones de pareja y la mentira entre jóvenes universitarios, lo que condujo a definir tres variables de opción múltiple: 1) la variable que evalúa la intensidad de la molestia percibida por el encuestado ante cada uno de las situaciones plasmadas en los quince ítems; 2) la variable que aborda la conducta inmediata del encuestado al darse cuenta de la situación, mensaje o hecho contenido en cada uno de las situaciones y 3) la indagación sobre la conducta asumida en la relación de la pareja pasado un tiempo después del reconocimiento de la situación. En el Anexo 1 se presenta el cuestionario utilizado.

Como el interés de este artículo es el estudio de la validez del instrumento elaborado para medir el nivel de intensidad de molestia que sufren los miembros de una pareja juvenil cuando el otro incurre en acciones mentirosas, nos referiremos exclusivamente a la primera variable mencionada.

\section{Procedimiento}

La investigación se desarrolló en tres fases: la primera, se centró en la revisión de publicaciones científicas relacionadas con el tema y consultas especializadas a expertos con el propósito de fijar alternativas metodológicas y procedimentales adecuadas al objeto de estudio; la segunda fase, se concentró en el diseño, validación interna y aplicación 
del instrumento; y, la tercera, se dirigió al análisis e interpretación de los resultados y conclusiones.

\section{Análisis de datos}

Se inicia el análisis de los resultados verificando las hipótesis de unidimensionalidad de las medidas, para luego continuar con el estudio de la eficacia de las categorías de respuesta de los ítems y con el estudio de la confiabilidad. Finaliza este apartado con el análisis del ajuste de los datos al modelo Rasch y la interpretación de las medidas estimadas utilizando el mapa de Wright. Para el análisis Rasch se utilizó WINSTEPS Versión 3.92 (Linacre, 2016).

\section{Resultados}

\section{Unidimensionalidad}

Verificar la unidimensionalidad del test hace relación a un hecho clave en el diseño de los test, como es que se construyen para medir una sola característica o un solo constructo. El análisis que se presenta a continuación va a permitir identificar los ítems dirigidos a un solo objetivo y a identificar, si existen, los que estén midiendo otra dimensión o característica.

El análisis de la dimensionalidad de la escala se realiza en dos pasos: Primero, se estiman las medidas de la escala usando el modelo Rasch, luego se realiza un análisis de componentes principales (ACP) de los residuos del modelo para identificar la existencia de subdimensiones sustanciales dentro de los ítems. En la Tabla 1 se muestran los resultados del ACP para los residuos estandarizados de las medidas obtenidas al ajustar el modelo Rasch.

El valor propio (eigenvalue) de la varianza no explicada en el primer contraste es 1.7319 equivalente a menos de 2 ítems que es el mínimo que se podría considerar para una segunda dimensión, adicional al hecho de que representa solamente el 7.6\% de la varianza no 
explicada que es algo menos que la cuarta parte de la varianza explicada de las medidas que es 33.9\% (Embretson y Reise, 2000).

\section{Tabla 1}

Item: Dimensionality

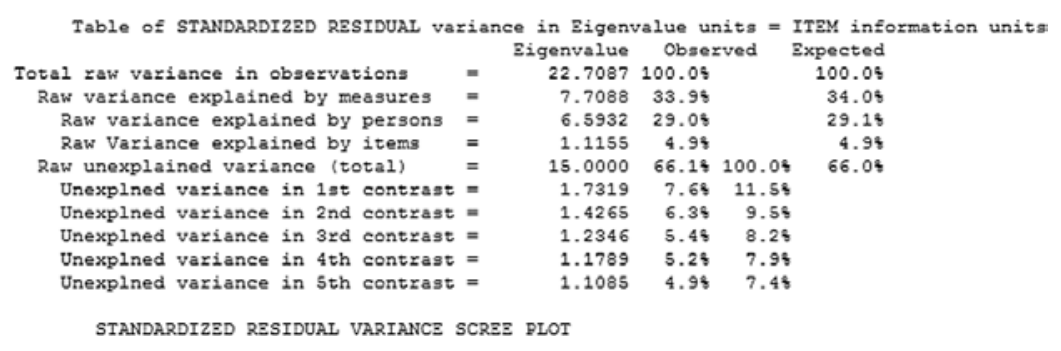

Fuente: Salida de Winsteps ${ }^{\oplus}$ (Version 3.92.0)

En consecuencia, no existen razones sólidas para negar la unidimensionalidad del test (Linacre, 2005). El análisis del primer contraste permitió identificar a los ítems: 5 (su pareja desconfió de usted) y 14 (su pareja no le creyó algo que usted le dijo) como los más alejados, ambos asociados con la confianza en la pareja, tal vez debido a que las expectativas de conducta frente a estas situaciones son moralmente inadmisibles en el ejercicio de las costumbres y rituales de seducción de la pareja, además que la confianza se asume como pilar fundamental en la relación (Núñez et al., 2015).

\section{Análisis de las categorías de respuesta}

Garantizada la unidimensionalidad del test, procedimos a verificar la validez de todas las categorías de respuesta introducidas: 1, 2, 3, 4, 5 . Este análisis se centraliza en observar la separación que existe entre las diferentes categorías para cada ítem, lo cual requiere calcular el umbral entre categorías (ver ecuación (1)) y su error de estimación.

Cuando dos umbrales consecutivos no tenían una diferencia entre ellos igual o superior al doble de la suma de sus errores estándar (intervalo de confianza al 95\%: 2 desviaciones estándar) se consideró que las dos categorías se solapan y por tanto se pueden consolidar en una sola. 
Análisis de las propiedades psicométricas de un cuestionario para medir la intensidad...

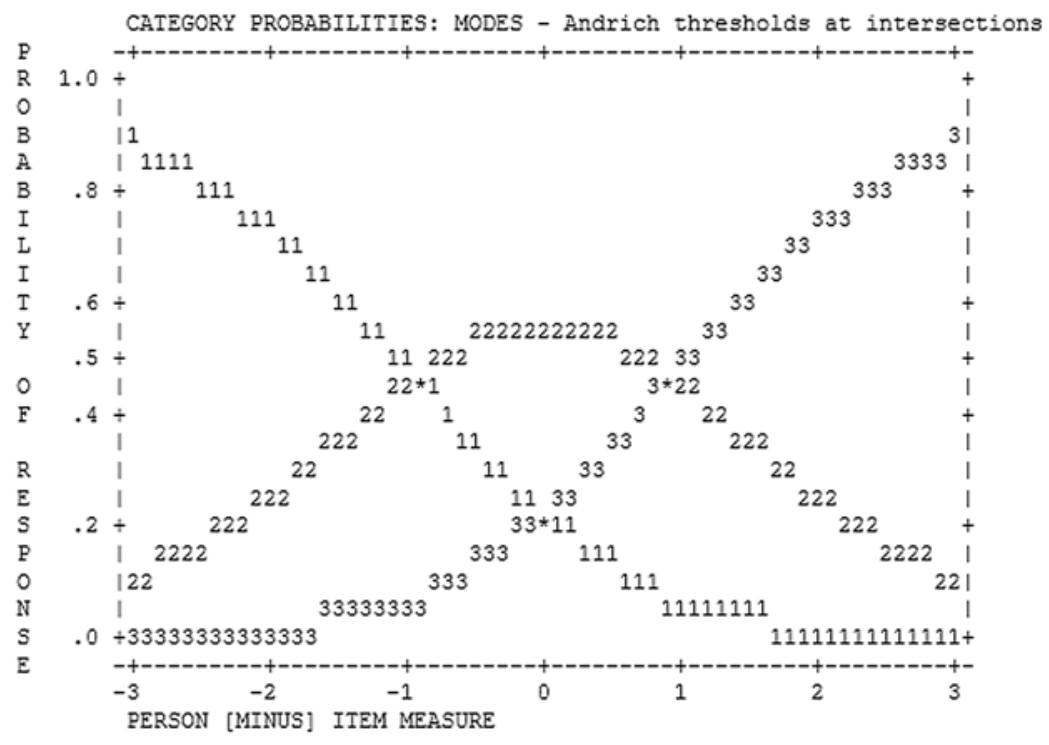

Figura 1. Category Probabilities: Modes - Andrich thresholds at intersections

Al aplicar este criterio y otros como son: el número de personas que respondieron en cada una de las categorías, el orden creciente en los niveles de sus medidas, Outfit menores que 2.0, la coherencia entre Medidas y Categorías (Linacre, 2002) se llegó a la conclusión de que la mejor escala solo requería de tres categorías: una categoría1, que podríamos llamar de nivel bajo de intensidad, como resultado de juntar las categorías 1 y 2 previas; una categoría 2, que podríamos catalogar de nivel medio de intensidad, como resultado de unir las categorías 3 y 4 y una categoría 3, que podríamos catalogar de nivel alto, correspondiente a la 5 del test original. Esta reducción mejoró ostensiblemente la separación de las categorías como se evidencia en la Figura 2. El agrupamiento de categorías condujo a ampliar el rango de las medidas de los ítems sin alterar la confiabilidad.

Adicionalmente, el agrupamiento hizo que la coherencia entre las medidas y las categorías se elevara a niveles moderadamente altos. 
La coherencia Medida (M) implica Categoría (C) que hace referencia a que dada una cierta diferencia entre la habilidad del individuo y la dificultad del ítem se puede predecir cuál es la categoría más probable de ser seleccionada por el individuo, se presenta alta: 66\%, 52\% y $74 \%$ para las categorías 1, 2 y 3 respectivamente. De otro lado la coherencia $\mathrm{C}$ implica $\mathrm{M}$, es decir, que dada la categoría seleccionada del individuo se puede predecir la diferencia entre su habilidad y la dificultad del ítem, tiene valores $31 \%$, $82 \%$ y $44 \%$ para las categorías 1,2 y 3 respectivamente. Salvo la categoría 1 que presenta un valor inferior a $40 \%$, la coherencia lograda es aceptable (Linacre, 2002).

\section{Confiabilidad}

Una característica psicométrica importante que deben poseer los tests es la confiabilidad. Alta confiabilidad en las medidas de los ítems (personas) significa que hay una alta probabilidad de que los ítems (personas) estimados con altas medidas realmente tengan medidas más altas que los ítems (personas) estimados con bajas medidas. La confiabilidad en las medidas de las personas y de los items depende de la variabilidad de las medidas y del error de estimación de ellas, y se hace mayor cuando se tiene un rango amplio de medidas y bajos errores de estimación. Como una medida de la calidad de las medidas, se calculan los índices de confiabilidad y separación tanto para los items como para las personas. El índice de confiabilidad en el modelo Rasch no es otra cosa que el índice de Cronbach que corresponde a las medidas Rasch, donde es posible calcular los errores de estimación para todos los items y todas las personas y se calcula como el cociente entre la varianza verdadera y la varianza observada, siendo la varianza verdadera la diferencia entre la varianza observada y la media de los errores de estimación, tanto para las personas como para los ítems.

Como se muestra en la Tabla 2, Winsteps reporta 0.96 para el índice de confiabilidad de los items, y un índice de separación de 4.91 que indica que existen 7 estratos de ítems separados por tres desviaciones estándar; el índice de confiabilidad de las personas es 0.77 y el índice de separación es de 1.84 lo que da lugar a 3 estratos separados por 3 desviaciones estándar. La separación de los ítems es indicativa 
de una muy buena estimación y de su capacidad de medir distintas intensidades de la molestia a la mentira. No es el caso para las personas cuyas medidas de intensidad de la molestia a la mentira son mucho más homogéneas, es decir, sus niveles de molestia no son tan disímiles, se clasifican apenas en tres grupos: bajo, medio y alto de acuerdo a la intensidad de la molestia sentida que se corresponden con las tres categorías de respuesta finalmente aceptadas para cada ítem.

\section{Tabla 2}

\section{Medidas básicas del modelo Rasch asociadas a la variable intensidad}

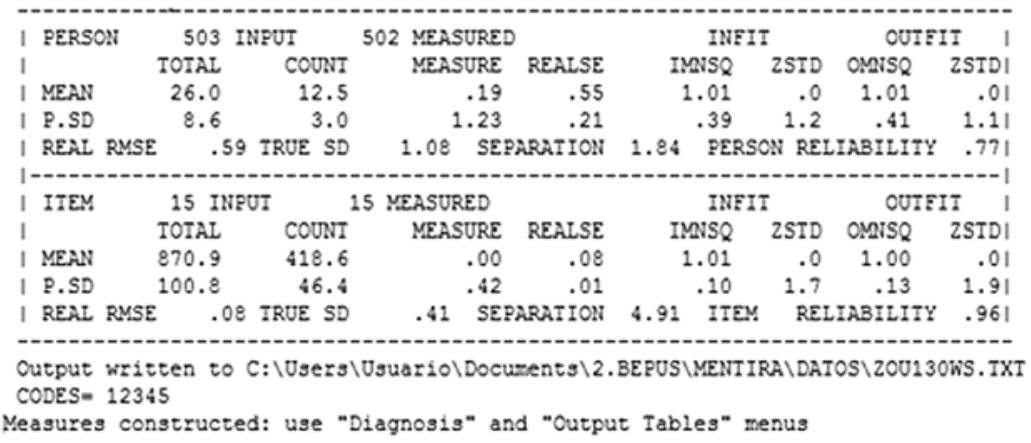

\section{Ajuste de los datos del modelo y validez}

Las ventajas del modelo de Rasch solo pueden ser obtenidas si los datos empíricos se ajustan al modelo. El ajuste del modelo se evalúa con base en la diferencia que existe entre el valor esperado por el modelo y el dato empírico, diferencia que se conoce como residuo. El debido tratamiento de estas diferencias permite detectar la presencia de respuestas aberrantes como son aquellas en que personas con poca cantidad de molestia a la mentira seleccionen categorías altas en items con alto nivel de molestia y viceversa, personas con mucha molestia seleccionen niveles bajos de molestia en ítems con bajos niveles de molestia.

El tratamiento de los residuos conduce a definir los estadísticos Outfit e Infit que son la estandarización de los residuos al cuadrado, ponderados por la varianza en el caso del Infit. Estos estadísticos tienen 
1.0 como valor esperado pero para efectos de medir la calidad del ajuste se considera que pueden oscilar, en nuestro caso, entre .7 y 1.4 ; valores menores que 7 reflejan poca aleatoriedad, típico de personas que responden siguiendo un patrón que no responde a sus sensaciones; los valores superiores a 1.4 , por el contrario, denotan demasiado variabilidad y son propios de las respuestas sorprendentes (Smith, Schumaker y Bush, 1998; Bond y Fox, 2001).

En la Tabla 3 se muestran los valores medios del Infit y del Outfit tanto para los ítems como para las personas lo que da cuenta de un ajuste general de los datos al modelo, ítems y personas. La tabla incluye, además, la desviación estándar y los valores mínimo y máximo.

\section{Tabla 3}

Valores del Infit y del Outfit para personas e items

\begin{tabular}{lllllllll}
\hline \multirow{2}{*}{ PARÁMETROS } & \multicolumn{4}{c}{ Infit MNSQ } & \multicolumn{4}{c}{ Outfit MNSQ } \\
& $M$ & $D E$ & Mín & Máx & $M$ & $D E$ & Mín & Máx \\
\hline Personas & 1.01 & .39 & .14 & 2.54 & 1.01 & .41 & .46 & 3.51 \\
Ítems & 1.01 & .10 & .87 & 1.25 & 1.00 & .13 & .86 & 1.30 \\
\hline
\end{tabular}

Los valores para el Infit y el Outfit de los items dan prueba de su buen ajuste al modelo, sus valores oscilan entre .86 y 1.30 . No obstante, los ítems 6 (su pareja no contestó al teléfono cuando usted lo llamó) y 10 (su pareja ocultó algo que usted debía saber) presentaron valores ZSTD 2.7 y -2.3 respectivamente, lo que obliga a reconsiderarlos en futuras aplicaciones del test. Los valores ZSTD son el resultado de una transformación realizada sobre los valores Infit y Outfit y que tiene una distribución $t$, que para muestras relativamente grandes, como la presente, permiten asumirla como normal estándar. El interés de estos valores es que sus propiedades distribucionales son más consistentes con respecto a la variación del tamaño muestral, razón por la cual es pertinente tenerlos en consideración (Smith, 2004).

Respecto a las personas, aunque ahora las desviaciones son bastante superiores a las obtenidas con los items, los valores medios son muy 
cercanos a 1.0, que es el valor esperado de ambos estadísticos, lo que permite afirmar el buen ajuste global por parte de las personas. Sin embargo, al analizar los valores de estos estadísticos en cada estudiante se observa que cerca del $10 \%$ de ellos tienen ajustes deficientes (las que poseen valores superiores a 1,4 ) o demasiado predecibles (valores menores a 0.7 ).

En la Tabla 4, se muestran las frecuencias absolutas y relativas de estudiantes para cada uno de los casos posibles de desajuste. Solo se consideraron los casos de estudiantes que superan los dos criterios considerados, esto es, los valores Infit (Outfit) y sus correspondientes ZSTD. Sin embargo, no se considera necesario suprimirlos del análisis ante los buenos resultados presentados por los items, aspecto que se reconoce de mayor relieve para el presente trabajo.

\section{Tabla 4}

Frecuencias absolutas y relativas para personas

\begin{tabular}{cccccc} 
INFIT & ZSTD & No. estudiantes & OUTFIT & ZSTD & No. estudiantes \\
\hline$<0.7$ & $<-2.0$ & $25(5 \%)$ & $<0.7$ & $<-2.0$ & $24(4.8 \%)$ \\
$>1.4$ & $>2.0$ & $28(5.6 \%)$ & $>1.4$ & $>2.0$ & $26(5.2 \%)$ \\
\hline
\end{tabular}

Ya con argumentos sólidos a favor del ajuste del modelo y haciendo uso de la propiedad de la medición conjunta se obtiene una representación geométrica (Figura 3) de las estimaciones de las medidas de los ítems y de las personas que respondieron el cuestionario. Esta representación, que se conoce como el mapa de Wright, permite observar la distribución de los ítems y de las personas simultáneamente de acuerdo a sus niveles de molestia a la mentira: en los ítems la intensidad que miden y en las personas la intensidad que sienten. Los ítems están ordenados de menor a mayor contenido de molestia, siendo el I_9 (su pareja le fue infiel) el de mayor molestia.

De acuerdo a la Tabla 2, la media de la medida de intensidad en los individuos es .19, ligeramente superior a la media .0 que por defecto tienen los ítems. Esta diferencia indica que la molestia sentida por las personas de la muestra es prácticamente igual a la molestia que 
en promedio miden los ítems, aspecto que refuerza la validez del test. No existen argumentos fuertes para negar la distribución normal de las medidas de la intensidad en los individuos (Kolmogorov p-valor: .27), comportamiento que permite realizar análisis estadísticos paramétricos asociados a estas medidas y que serán objeto de un próximo trabajo.

Prácticamente el $50 \%$ de las medidas de las personas están en el mismo intervalo de las medidas de los ítems: $[-1.13, .55]$ dando a entender que el cuestionario apunta a los valores centrales de la muestra de estudiantes que se corresponden con los valores medios del nivel de intensidad (el 44\% de las respuestas se clasificaron en la categoría 2 de la intensidad de la molestia y tienen una media en las medidas de .07 que se corresponde bastante bien con la de los ítems).

Ahora bien, como los ítems tienen tres opciones de respuesta que hemos definido como baja, media y alta, cuya selección se asocia con diferentes niveles de intensidad de la molestia, se logra un recorrido mayor en los niveles de molestia que pueden captar los ítems, tal como se evidencia en la Figura 3.

En este mapa, se muestran las medidas de la intensidad de la molestia ante la mentira en las personas. A la derecha se encuentran las medidas de los ítems de acuerdo a la categoría seleccionada. Nótese que en cada ítem aparece tres veces a lo largo de la recta, cada una de ellas está asociada con las categorías 1, 2 y 3, mostrando el nivel de intensidad de la molestia a la mentira que miden las categorías de respuesta de cada ítem. Es así que si el ítem tiene la terminación .1 está indicando que la probabilidad de que un individuo con la misma medida en que está ubicado el ítem, tiene la misma probabilidad de seleccionar la categoría 1 o cualquier otra categoría superior; si termina en .2 es la misma probabilidad de seleccionar la categoría 2 o superior; y si termina en .3 tiene la misma probabilidad de seleccionar la categoría 3 o alguna inferior. No obstante que se siguen presentando los vacíos entre el ítem 9 y los demás ítems, el espectro de valores para la intensidad de los ítems de acuerdo a sus categorías aumenta considerablemente, va de -3.2 a 2.5 lógitos.

Dado que el ítem 9 (su pareja le fue infiel) está muy alejado de los demás ítems, debilitando la validez del test, se hace necesario construir 
ítems cuyo contenido de molestia sea más cercano al producido por el ítem 9 y que ayuden a medir mejor la intensidad de la molestia de las personas con valores semejantes. En el intervalo $[-.44, .55]$ se encuentran $34 \%$ de los estudiantes de la muestra que se puede afirmar fueron bastante bien medidos por el instrumento creado.

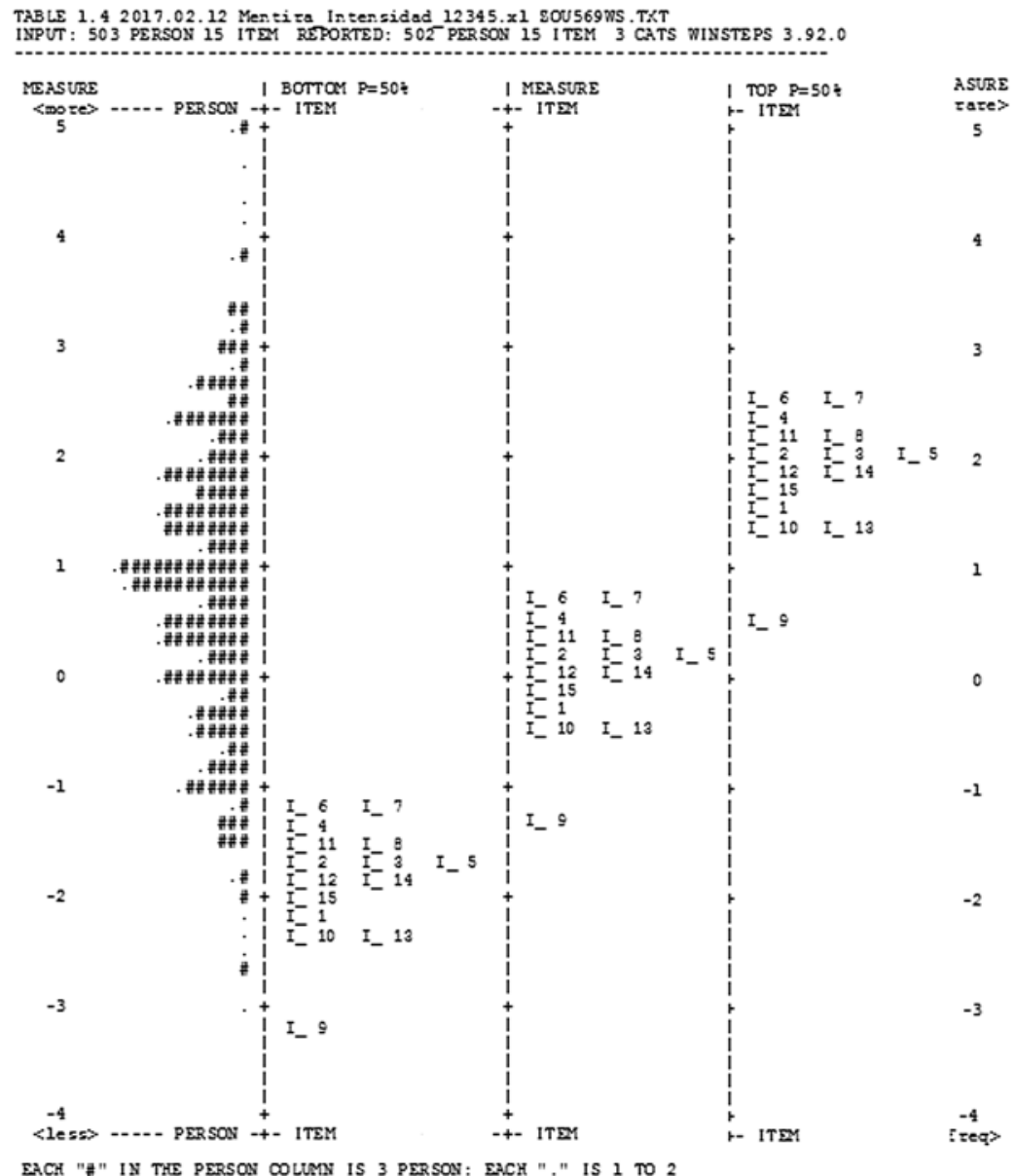

Figura 3. Mapa de Wright para los ítems y sus categorías. 


\section{Discusión}

El análisis realizado sobre el test para medir la intensidad de la molestia ante la mentira utilizando el modelo Rasch nos permite obtener las siguientes conclusiones:

En primer lugar, se mostró que no existe suficiente evidencia para negar la unidimensionalidad del test, es decir, el test efectivamente mide el constructo en estudio, como es, la intensidad de la molestia a la mentira.

El ajuste entre el nivel de la intensidad de las personas y la de los ítems es muy alto, lo que afirma la validez del test que se puede considerar ajustado para medir poblaciones semejantes.

Es suficiente que la escala de respuesta para la intensidad de la molestia en cada situación contenga solo tres niveles: bajo, medio y alto. Con solo estos tres niveles se aumenta tanto el ajuste de los datos al modelo, como se amplía suficientemente el rango de la intensidad que miden estos ítems, haciendo que el cuestionario permita medir con suficiencia la magnitud de la intensidad de la molestia de las personas a las que se aplique y que sus índices de consistencia Categoría Medida sean relativamente altos. Esta categorización de las respuestas y la buena correspondencia evidenciada entre los ítems y los estudiantes permite también clasificarlos en tres categorías en términos de su intensidad de molestia a la mentira: baja, media y alta que guardan una estrecha correspondencia con las mismas categorías de los ítems.

Los valores incite y outfit evidencian el buen ajuste de los datos al modelo Rasch garantizando la alta validez de los reactivos del test (Smith, 2004). Sin embargo, la validez se puede aumentar construyendo más ítems que abarquen un espectro mayor de la intensidad de la molestia ocasionado por la mentira, en particular con intensidades menores que la experimentada por el ítem 9 (su pareja le fue infiel) y superiores a la del ítem 13 (su pareja contó un secreto de ustedes dos).

La confiabilidad de las medidas de los ítems es alta (.98), de lo cual se sigue que el orden de la dificultad de los ítems se conservará cuando se aplique el cuestionario a otras muestras similares. 
Igualmente, la confiabilidad o consistencia de las medidas de los examinados también es alta (.77) indicativo de que es altamente probable que el orden de la molestia de los individuos pueda ser replicado con la aplicación de un test semejante.

En consecuencia, se recomienda su uso para el estudio de esta variable social, molestia ante la mentira de la pareja heterosexual, con el cual se busca una mayor comprensión y explicación de esta compleja relación de pareja.

\section{Referencias}

Andrich, D. (1978). A rating formulation for ordered response categories. Psychometrika, 43, 561-573. https://doi.org/10.1007/ BF02293814

Andrich, D. (1988). Rasch models for measurement. Newbury Park, CA: Sage. https://doi.org/10.4135/9781412985598

Ayala, A., Rodríguez-Blázquez, C., Frades-Payo, B., João Forjaza, M., Martínez-Martín, P., Fernández-Mayoralase, G. y Rojo-Péreze, F. (2012). Propiedades psicométricas del Cuestionario de Apoyo Social Funcional y de la Escala de Soledad en adultos mayores no institucionalizados en España. Gaceta Sanitaria, 26(4), 317-324. https://doi.org/10.1016/j.gaceta.2011.08.009

Bond, T.G. y Fox, C.M. (2001). Applying the Rasch model: fundamental measurement in the human sciences. Mahwah, NJ: LEA. https:// doi.org/10.4324/9781410600127

Botero Álvarez, S., Builes Cedula, C. F., García-Bonilla, Z., GilSaldarriaga, M. y Ramírez-Guerrero, K. (2016). Las creencias en el noviazgo universitario: infidelidad, mentira y engańo. Funlam Journal of Students' Research, (1), 42-51. https://doi.org/ $10.21501 / 25007858.2138$

Embretson, S.E. y Reise, S.P. (2000). Item response theory for psychologists. Mahwah, NJ: LEA. 
Hambleton, R. K., Swaminathan, H. y Rogers, H. J. (1991). Fundamentals of item response theory. Beverly Hills, CA: Sage. Linacre, J. M. (2002). Optimizing Rating Scale Category Effectiveness. Journal of Applied Measurement, 3(1), 85-106.

Linacre, J. M. (2005). Winteps ${ }^{\circ}$ Rasch Measurement Computer Program. Chicago, IL: Winsteps.com.

Linacre, J. M. (2016). Winsteps ${ }^{\circledR}$ (Version 3.92.0) [Computer Software]. Beaverton, OR: Winsteps.com. Available from http://www. winsteps.com/

Núñez, F., Cantó-Milà, N. y Seebach, S. (2015). Confianza, mentira y traición: El papel de la confianza y sus sombras en las relaciones de pareja. Sociológica (México), 30(84), 117-142. Recuperado en 24 de diciembre de 2016, de http://www.scielo.org.mx/scielo. php?script=sci_arttext\&pid=S0187-01732015000100004\&lng $=\mathrm{es} \& \mathrm{t} \operatorname{lng}=\mathrm{pt}$.

Pozueco-Romero, J. M., Moreno-Manso, J. M., Blázquez-Alonso, M. y García-Baamonde, Ma . E. (2013). Psicópatas integrados/ subclínicos en las relaciones de pareja: perfil, maltrato psicológico y factores de riesgo. Universidad de Extremadura. Papeles del Psicólogo, 34(1), 32-48. Recuperado de: http://www.papelesdelpsicologo.es/pdf/2169.pdf

Prieto, G. y Delgado, A.R. (2003). Análisis de un test mediante el modelo de Rasch. Psicothema, 15(1), 94-100.

Rasch, G. (1977). On specific objectivity: An attempt at formalizing the request for generality and validity of scientific statements. En M. Glegvad (Ed.), The Danish Yearbook of Philosophy (pp. 59-94). Copenhagen: Munksgarrd.

Rodríguez, C., Martínez-Pecino, R. y Durán, M. (2015). Ciberacoso en la adolescencia y revelación de las agresiones. Apuntes de Psicologia, 33(3), 95-102.

Smith, Jr., E.V. (2001). Evidence for the reliability of measures and validity of measure interpretation: A Rasch measurement perspective. Journal of Applied Measurement, 2, 281-311. 
Smith, E.V. (2004). Evidence for the Reliability of Measures and Validity of Measure Interpretation: A Rasch Measurement Perspective. En E.V. Smith y R.M. Smith (Eds.), Introduction to Rasch Measurement (pp. 93-122). Maple Grove, MN: JAM Press.

Smith, R.M. (2004). Fit Analysis in Latent Trait Measurement Models. En E.V. Smith y R.M. Smith (Eds.), Introduction to Rasch Measurement (pp. 73-92). Maple Grove, MN: JAM Press.

Smith, R.M., Schumacker, R.E. y Bush, M.J. (1998). Using item mean squares to evaluate fit to the Rasch model. Journal of Outcome Measurement, 2, 66-78.

Wright, B.D. y Stone, M.H. (1979). Best test design. Chicago, IL: Mesa Press.

Yánez, H., Ferrel, F.R., Ortiz, L. y Yáńez, G. (2017). Efectos de la mentira en las relaciones de pareja entre jóvenes universitarios heterosexuales. Revista Psicología desde el Caribe, 34(1), 71-90.

Recibido: 3 de junio, 2017

Revisado: 25 de junio, 2018

Aceptado: 1 de abril, 2019 


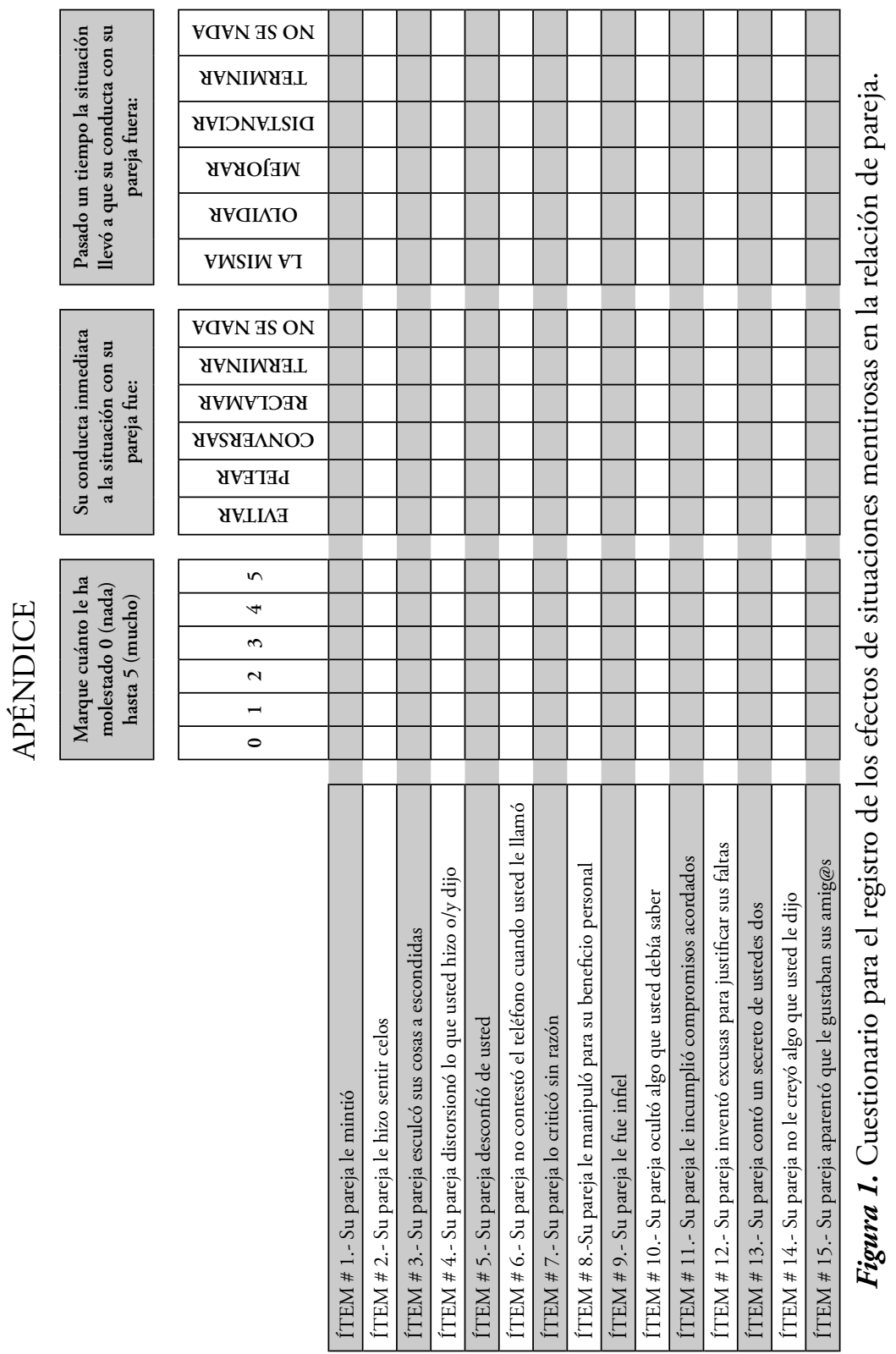

